\title{
Effects of Synchronization Errors on Wavelet-based CR-OFDM Systems in Doubly Selective Fading Channels
}

\author{
ARJUN KETHUPURA RANGASWAMY ( $\nabla$ arjunkrmys@gmail.com ) \\ Vidyavardhaka College of Engineering https://orcid.org/0000-0002-7640-4111 \\ Surekha T P \\ Vidyavardhaka College of Engineering
}

\section{Research Article}

Keywords: Bit Error Rate (BER) , Cognitive Radios (CR) , Multi-Carrier Modulation (MCM) , OFDM , WAVELETS

Posted Date: July 1st, 2021

DOI: https://doi.org/10.21203/rs.3.rs-580417/v1

License: (c) (i) This work is licensed under a Creative Commons Attribution 4.0 International License.

Read Full License 


\title{
Effects of synchronization errors on Wavelet-based CR-OFDM systems in doubly selective fading channels
}

\author{
Arjun K R • Surekha T P
}

Received: date / Accepted: date

\begin{abstract}
The major setback of a Multi-Carrier Modulation (MCM) is Synchronization Errors, which includes time, frequency and phase offset. Especially, Wavelet based MCM catches the eyes of researchers due to its flexibilities which are seen as one of the strong contender for Cognitive Radios. Synchronization errors are mainly due to mobility between nodes and suboptimal local oscillators and it is necessary to learn the behaviour of wavelets under these channel fading conditions. In this paper, we present the joint effects of Wavelet-based Cognitive Radio OFDM (CR-WOFDM) systems under Synchronization Error in terms of based bit error rate (BER). BER Outputs of WOFDM is compared with FFT based OFDM with Cyclic Prefix (CPOFDM) systems in a doubly-selective channel by designing a communication system for computer simulation. Several well-known wavelets are chosen and analysed, including Daubechies (db), Symlets (sym), Coiflets (coif), FejérKorovkin (fk) filters, and biorthogonal (bior) wavelets. First, we show the behaviour of wavelets in terms of BER by considering different doubly selective channel Power Delay Profile (PDP) like, AWGN, FLAT, Pedestrian and Vehicular and channel Doppler models like, Uniform and JAKES. Finally, we calculate and plot Signal-to-Interference Ratio (SIR) of WOFDM with Time and Frequency Offset and compared the results with FFT based CP-OFDM.
\end{abstract}

\footnotetext{
Arjun K R

Vidyavardhaka College of Engineering

Department of Electronics and Communication,

Gokulam, Mysore, India

Tel.: +91-9632102676

E-mail: arjunkrmys@gmail.com

Surekha T P

Vidyavardhaka College of Engineering

Department of Electronics and Communication,

Gokulam, Mysore, India
} 
Keywords Bit Error Rate (BER) - Cognitive Radios (CR) · Multi-Carrier Modulation (MCM) · OFDM · WAVELETS

\section{Introduction}

To keep up with the growing demand for high data rates, which in-turn to efficiently utilize the available spectrum, Cognitive Radio (CR) is a promising technology. Primary function of CR systems is to access unused spectrum dynamically which is originally allocated to user known as Primary User (PU) and same spectrum will be reallocated to Secondary User (SU) [13]. Information in wireless communications is transmitted by radiating a modulated electromagnetic wave. Most of the current day communication employs multi carrier modulation (MCM) to meet increasing need of higher bandwidth [15]. MCM techniques used in CR systems include Orthogonal Frequency Division Multiplexing (OFDM), Wavelet Packet Modulation (WPM) or Wavelet-Based OFDM (WOFDM).

The sinusoid basis of OFDM has been replaced with Wavelet basis leading to WOFDM. The flexibility of wavelets to adapt to channel conditions makes WOFDM as a best choice for next generation communication systems [9]. The data from MCM are up converted at a certain carrier frequency by means of a transmit antenna and then transmitted in a channel, which is often a timedispersive environments [6]. The energy of this, time-disrupted data is then picked up by means of a receive antenna. During this process of transmission and reception of data through time-disruptive channel, the properties of original data changes and multiple copies of transmitted data arrives at receiver resulting in severe synchronization errors.

In-spite of attractive features, Wavelet-based OFDM still suffers severely during signal detection over multi-path fading channels [4]. Estimating symbol timing offset, especially in a doubly selective fading channel plays a vital role to maintain orthogonality in WOFDM where Cyclic Prefix (CP) is not present. For systems with MCM, there are three types of channel estimation and synchronization methods [2]; Firstly, The blind approach improves spectral efficiency but is less used in most practical applications because for accurate estimation it requires a longer observation window and limits their tracking capability by avoiding the overhead of training sequences. Second method being, Semi-blind methods which only require the transmission of a small number of parameters to solve estimation uncertainty and they offer a useful tradeoff between spectral efficiency and estimation accuracy. Finally, to boost the estimation performance, the pilot-based synchronization and estimation is implemented while incurring a tolerable training symbol overhead.

The remainder of the paper is laid out as follows, Literature review of various authors related to MCM, CR, OFDM, WOFDM and different channels are presented in section 2 . Section 3 provides the brief modeling of synchronization errors in OFDM and WOFDM. The communication system designed 
for this study and computer simulation methodology is presented in section 4 . Paper is concluded with results, discussions and conclusion in section 5 and 6 .

\section{Literature Review}

S. K. Sharma et al. [16] in his survey paper discussed the importance of CR to overcome spectrum scarcity problem in future wireless communications under practical imperfections. Several imperfections like amplifier non linearity, multi-carrier distortion, channel imperfections, synchronization errors like frequency offset and phase offset due to transmitter and receiver local oscillators (LO), and time offsets due to multi-path fading has been addressed.

For OFDM-based Cognitive Radio Network, signal detector and synchronization parameter estimators are combined in $[10,5]$. Author presented a minimum cost detection scheme in multipath fading channel for cognitive radio. It is found that, the diversity gain of primary user has been increased by employing the blind synchronization algorithm for estimation of symbol time offset (STO) and carrier frequency offset (CFO). It has been discovered that as the PU's spectrum occupancy likelihood decreases, the SU's effective throughput rises even when the Cognitive Radio OFDM system operating under frequencyselective fading channels.

For OFDM/FBMC, an improved joint estimation method has been proposed for sampling time-offset; carrier frequency offset, and channel impulse response [3]. Under the assumptions of Gaussian noise and independent input symbols, the proposed joint ML estimator employs a maximum-likelihood (ML) estimation of the unknown parameters based on pilot data. As a performance benchmark in the ML sense, the Cramer-Rao bound on the covariance matrix of unbiased estimators of the joint parameter vector under consideration is determined.

Nasir et al. in [14] discussed the time and frequency synchronization as an primary requirement in any wireless communication systems and author provides detailed review on synchronization for a specific time period. In singleinput single-output (SISO), multiple-input multiple-output (MIMO), cooperative relaying, and multiuser interference networks, the author performed a systematic study and classification of recent research developments in timing and carrier synchronization.

Authors in [8] W. Guo et al. holistically investigated the effects of synchronization during channel impairments conditions on Known Interference (KI) cancellation. To begin, the normalized time synchronization error is modeled using inter-symbol interference (ISI), while the frequency synchronization error and phase noise are modeled using inter-frequency interference (IFI). The closed form formulation of the KI cancellation ratio (KICR) is then calculated for the multi-path block fading channel, taking into consideration imperfect time-frequency synchronization and channel estimation, as well as phase noise. Author's theoretical analysis is supported by simulation findings, where the simulation result shows KICR is more vulnerable to phase noise in compari- 
son to time-frequency synchronization and channel estimation errors. Furthermore, it has been demonstrated that a lower KI power is less vulnerable to implementation flaws.

H. Abdzadeh-Ziabari et al [1] suggested a new approach for joint timing, frequency offset, and multipath fast fading channel estimation. The new scheme was created by combining the Maximum-Likelihood (ML) criterion, the transmission of a preamble, and the channel's Basis expansion Modeling (BEM). A new scheme for reducing joint estimation complexity was also proposed, which was based on a low-complexity CFO estimation algorithm. Using MSE, it was demonstrated that the new method significantly improves time, frequency offset, and channel estimation performance when compared to previously published methods. The work in this paper has been extended to uplink OFDM and briefed in [2].

The author proposed a novel algorithm for time and frequency synchronization in uplink OFDM using a Doubly Selective Channel (DSC) and a Basis Expansion Model (BEM) to represent a DSC in [2]. A maximum likelihood (ML) approach is proposed to jointly estimate the timing and carrier frequency offsets of various users, as well as the BEM coefficients. Using a space-alternating generalized expectation-maximization algorithm, the maximization problem for all users is then broken down into several smaller maximization problems for a single user. The proposed scheme's computational complexity, implementation issues, and mean square error are all assessed. The author also demonstrates that the current scheme improves performance significantly.

An Investigation into Wavelet Packet Modulation's Sensitivity to Time Synchronization Error is performed on various standard wavelets in [12] and compared with FFT based OFDM systems. Authors performed computer simulation by designing a communication system with DPSK modulation, AWGN channel, and 128 orthogonal subcarriers leading to 7 stages of wavelet packet tree. Several well-known wavelets like Daubechies, Symlets, Coiflets, discrete Meyer, and biorthogonal wavelets are used and analyzed. Further, author claims that minor timing differences between transmitter and receiver have a big impact on WPM compared to classic OFDM systems.

\section{Synchronization Errors in OFDM and WOFDM}

The term channel characterization refers to the process of determining whether a channel is frequency or time selective. Oscillator drifts, as well as motion between transmitter and receiver, can cause channels with time variations within the channel. Doubly-selective channels have a response that is both time and frequency selective. These effects lead to Synchronization errors in any communication systems [7]. Below section provides the modeling of OFDM and WOFDM systems under doubly-selective channels. 


\subsection{Frequency offset error}

Frequency offsets are usually caused by a minute frequency mismatch between the transmitter and receiver's local oscillators. The Doppler change is calculated as follows [12]:

$$
\mathcal{F}_{d k}=\frac{v_{r} \mathcal{F}_{k}}{C} \cos \alpha
$$

Where, $\mathcal{F}_{d k}$ in equation (1) is the Doppler frequency shift, $\mathcal{F}_{k}$ is the subcarrier frequency, $\alpha$ is the velocity vector angle, $\mathrm{C}$ is speed of light and $v_{r}$ is the difference in speed between the transmitter and the receiver. The main carrier frequency $\mathcal{F}_{c}$ and the baseband sub-carrier frequency $\mathcal{F}_{s c}$ can be used to determine the frequency of each sub-carrier $\mathcal{F}_{k}$, and can be represented as,

$$
\mathcal{F}_{k}=\mathcal{F}_{s c} \pm \mathcal{F}_{c}
$$

The relative frequency offset $\Delta_{f}$ which is the ratio of frequency offset and sub-carrier spacing can be expressed with the help of (1) and (2),

$$
\Delta_{f}=\frac{v_{r}}{C} \cos \alpha
$$

\subsubsection{Frequency offset error in OFDM}

At the receiver, the frequency offset can be represented by multiplying the received signal in time by a complex exponential with a frequency component equal to the frequency offset value. The relationship between the transmitted $\mathcal{S}[n]$ and received signals $\mathcal{R}[n]$ under the influence of frequency offset can be expressed as follows [19]:

$$
\mathcal{R}[n]=\mathcal{S}[n] e^{\frac{j \pi \Delta_{f n}}{N} \phi_{0}}+w[n]
$$

Where $\mathrm{N}$ in equation (4) represents total number of sub-carriers, w[n] denotes AWGN channel noise and $\phi_{0}$ is the initial phase. Under the frequency offset $\Delta_{f}$ at the receiver, the FFT output corresponding to the $K^{\text {th }}$ sub-carrier can be expressed as:

$$
\begin{gathered}
\hat{a}_{k^{\prime}}=\frac{1}{N} \sum_{n=0}^{N-1} \mathcal{R}[n] e^{-j 2 \pi \frac{k^{\prime} n}{N}} \\
\hat{a}_{k^{\prime}}=\frac{1}{N} \sum_{n=0}^{N-1} a_{k} \sum_{k=0}^{N-1} e^{-j 2 \pi \frac{\left(k-k^{\prime}+\Delta_{f}\right) n}{N}} \\
\hat{a}_{k^{\prime}}=a_{k^{\prime}} \frac{\sin \left(\pi \Delta_{f}\right)}{N \sin \frac{\pi \Delta_{f}}{N}} e^{j 2 \pi\left(\frac{N-1}{N}\right) \Delta_{f}}+\frac{1}{N} \sum_{k=0}^{N-1} a_{k} \frac{\sin \left(\pi\left(k-k^{\prime}+\Delta_{f}\right)\right)}{\sin \left(\frac{\pi\left(k-k^{\prime}+\Delta_{f}\right)}{N}\right)} e^{-j 2 \pi \frac{\left(k-k^{\prime}+\Delta_{f}\right) n}{N}}
\end{gathered}
$$


The first component of equation (7) represents a useful demodulated signal that has been attenuated and shifted as a result of frequency offset. The intercarrier interference (ICI) term is found in the second part. However, AWGN channel noise is considered as $\mathrm{w}[\mathrm{n}]=0$ in equation $(7)$.

\subsubsection{Frequency offset error in WOFDM}

At the receiver of WOFDM and under a loss of frequency synchronization, the detected data for the $k^{t h}$ subcarrier and the $u^{t h}$ symbol can be expressed as $[12]:$

$\hat{a}_{u^{\prime}, k^{\prime}}=\sum_{u} \mathcal{R}[n] \xi_{l}^{k^{\prime}}\left[u^{\prime} N-n\right]=\sum_{u} \sum_{k=0}^{N-1} a_{u, k}\left(\sum_{n} \xi_{l}^{k}[n-u N] e^{j 2 \pi \Delta_{f}\left(\frac{n}{N}\right) \xi_{l}^{k}\left[u^{\prime} N-n\right]}\right)$

Where $\xi$ in equation (8) denotes wavelet packet duals, $u$ denotes the index of WPM symbols. The cross-waveform function $\Omega\left[\Delta_{f}\right]$ can be defined as:

$$
\Omega_{k, k^{\prime}}^{u, u^{\prime}}=\sum_{n} e^{j 2 \pi \Delta_{f}\left(\frac{n}{N}\right) \xi_{l}^{k}\left[u^{\prime} n-N\right]}
$$

The demodulated information bit of the $k^{t h}$ subcarrier and the $u^{\text {th }}$ WOFDM symbol that has been corrupted by interference due to orthogonality loss can be expressed as:

$$
\hat{a}_{u^{\prime}, k^{\prime}}=a_{u^{\prime}, k^{\prime}} \Omega_{k, k^{\prime}}^{u, u^{\prime}}\left[\Delta_{f}\right]+\sum_{u} a_{u^{\prime}, k^{\prime}} \Omega_{k, k^{\prime}}^{u, u^{\prime}}\left[\Delta_{f}\right]+\sum_{u} \sum_{k=0}^{N-1} a_{u^{\prime}, k^{\prime}} \Omega_{k, k^{\prime}}^{u, u^{\prime}}\left[\Delta_{f}\right]
$$

The first term in equation (10) refers to the valuable data that has been attenuated and rotated. The ISI attributable to symbols transmitted on the same sub-channel is given in the second term, and the ICI computed over the entire frame is given in the third term. The channel effects are not considered in above equation.

\subsection{Time offset error}

Time offset error in multi-carrier modulation occurs mainly due to misalignment of multi-carrier symbols at receiver. The obtained data samples are shifted to the left or right by a time offset value $\Delta_{t}$, depending on the sign of the $\Delta_{t}$, to model the time synchronization error. The presence of a time synchronization error is denoted as:

$$
R\left[n \pm \Delta_{f}\right]=S[n]+w[n]
$$

Where, $R[n]$ is the received signal, $S[n]$ is the transmitted signal and $w[n]$ is the channel noise. 


\subsubsection{Time offset error in OFDM}

OFDM utilizes low complexity yet effective approach termed as Cyclic Prefix (CP) to overcome synchronization errors. Neglecting the effects of channels $w[n]=0$, the timing error at receiver of OFDM after FFT can be represented as $[19,12]$ :

$$
\begin{array}{r}
\hat{a}_{u^{\prime}, k^{\prime}}=\frac{N-\Delta_{t}}{N} a_{u^{\prime}, k} e^{j 2 \pi \frac{\left.\left(k^{\prime}\right) \Delta_{t}\right)}{N}}+\frac{1}{N} \sum_{k=0}^{N-1-\Delta_{t}} \sum_{k=0}^{N-1} a_{u^{\prime}, k} e^{j 2 \pi \frac{k\left(n+\Delta_{t}\right.}{N}} e^{-j 2 \pi \frac{\left(k^{\prime}\right) n}{N}} \\
+\frac{1}{N} \sum_{n=N-\Delta_{t}}^{N-1} \sum_{k=0}^{N-1} a_{u+1, k} e^{j 2 \pi \frac{k\left(n-N+\Delta_{t}\right)}{N}} e^{-j 2 \pi \frac{\left(k^{\prime}\right) n}{N}(12)}
\end{array}
$$

First term in equation (12) indicates useful data which is phase shifted and attenuated. The second and third term gives ICI and ISI components respectively. Let us assume for the case when $\Delta_{t}<N_{c p}$. Where, Ncp number of cyclic prefixes symbols. The demodulated output is given by,

$$
\hat{a}_{u^{\prime}, k^{\prime}}=\hat{a}_{u^{\prime}, k} e^{-j 2 \pi \frac{\left(k^{\prime}\right) \Delta_{t}}{N}}
$$

\subsubsection{Time offset error in WOFDM}

Since the WOFDM does not use Cyclic Prefix, the position of the offset is irrelevant. Under time offset error $\Delta_{t}$, the demodulation process error can be calculated as:

$\hat{a}_{u^{\prime}, k^{\prime}}=\sum_{u} \mathcal{R}[n] \xi_{l}^{k^{\prime}}\left[u^{\prime} N-n+\Delta_{t}\right]=\sum_{u} \sum_{k=0}^{N-1} a_{u, k}\left(\sum_{n} \xi_{l}^{k}[n-u N] \xi_{l^{\prime}}^{k^{\prime}}\left[u^{\prime} N-n+\Delta_{t}\right]\right)$

Let, the term $\left(\sum_{n} \xi_{l}^{k}[n-u N] \xi_{l^{\prime}}^{k^{\prime}}\left[u^{\prime} N-n+\Delta_{t}\right]\right)$ in equation (14) represented as $\Omega_{k, k^{\prime}}^{u, u^{\prime}}\left[\Delta_{t}\right]$ to relieve complexity. Finally, the WOFDM symbol affected by time synchronization error be represented as:

$$
\hat{a}_{u^{\prime}, k^{\prime}}=a_{u^{\prime}, k^{\prime}} \Omega_{k, k^{\prime}}^{u, u^{\prime}}\left[\Delta_{t}\right]+\sum_{u} a_{u^{\prime}, k^{\prime}} \Omega_{k, k^{\prime}}^{u, u^{\prime}}\left[\Delta_{t}\right]+\sum_{u} \sum_{k=0}^{N-1} a_{u^{\prime}, k^{\prime}} \Omega_{k, k^{\prime}}^{u, u^{\prime}}\left[\Delta_{t}\right]
$$

The first term in equation (15) refers to the valuable data that has been attenuated due to time synchronization error. The ISI attributable to symbols transmitted on the same sub-channel is given in the second term, and the ICI computed over the entire frame is given in the third term. 


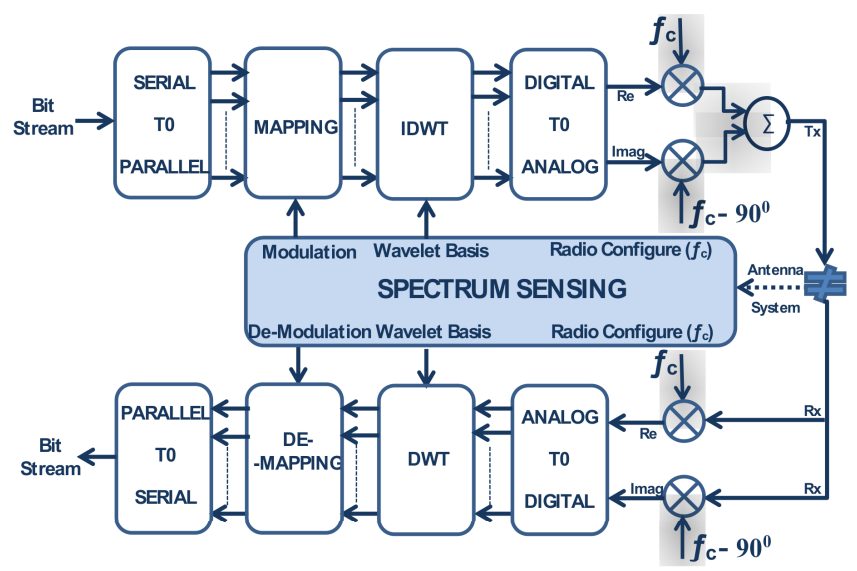

Fig. 1 System building Block of Wavelet Based CR OFDM system

\section{Methodology Used}

In-order to learn the abilities and the impact of Wavelets in multi-carrier modulation it is necessary to analyze the interference in Wavelet based CR OFDM system under the influence of Synchronization errors and doubly selective channels. Figure 1 shows the system block of Wavelet based Cognitive Radio (CR) OFDM system. The IDFT and DFT blocks in transmitter and receiver of OFDM are replaced with wavelet basis according the selection by feedback from Spectrum Sensing Block which is the fundamental building block of Wavelet based CR OFDM system shown in figure 1. Spectrum sensing block decides the modulation type, configures radio and selects the wavelet basis depending upon channel conditions.

The output of Digital to Analog converter will be a complex output. The complex symbol is then up converted by multiplying $\mathcal{F}_{c}=\cos \left(2 \pi f_{c} \frac{n}{N}\right)$ with real part and $\mathcal{F}_{c}-90^{0}=\sin \left(2 \pi f_{c} \frac{n}{N}\right)$ with Imaginary part before transmitting through an antenna. Received signal at the receiver is processed by first down converted by multiplying $\mathcal{F}_{c}=\cos \left(2 \pi f_{c} \frac{n}{N}\right)$ which results in real component and $\mathcal{F}_{c}-90^{\circ}=\sin \left(2 \pi f_{c} \frac{n}{N}\right)$ resulting in imaginary component. These data is passed through Analog to Digital Converter to process further. However, Low Pass Filters (LPF), Power Amplifiers (PA) and Low Noise Amplifiers (LNA) are not included in the block diagram.

In this paper, a combination of Bit Error Rate (BER) over Signal to Noise Ratio (SNR) doubly-selective channel for different wavelet basis like Daubechies (db), Symlets (Sym), Fejér-Korovkin filters (fk), Bi-Orthogonal (bior) are used and Signal to Interference Ratio (SIR) is calculated for Time and Frequency offset errors. The theoretical analysis of section 3 has been implemented for various mobility conditions between transmitter and receiver, various wavelet filter lengths, different Doppler models has been considered and compared with OFDM systems. The radio configuration for both OFDM 
and WOFDM is kept constant and for simplicity one-tap equalizer is used at the receiver.

\section{Results and Discussions}

In this section, the performance of various wavelets and with different filter sizes under synchronization error has been simulated. BER versus SNR and calculation of SIR with respect to Time offset error and Frequency offset error has been used as performance parameters. Parameters used for simulation is provided in Table I . These parameters are kept constant throughout the simulation.

The RMS, or root mean square, is a measure of the width of the power delay profile. RMS delay spread $\tau_{R M S}$ used in this study for simulation is defined as [17]:

$$
\tau_{R M S}^{2}=\frac{\int_{0}^{\infty}\left(t-T_{0}\right)^{2} P_{R}(t) d t}{\int_{0}^{\infty} P_{R}(t) d t}
$$

Where, $P_{R}(t)$ is Power Delay Profile (PDP) and $T_{0}$ is the mean delay. The time origin is chosen in such a way that $P_{R}(t)=0$ for $T_{0}<0$. Jake's channel $r(t)$ used can be modeled as [18]:

$$
r(t)=r_{i}(t)+j r_{q}(t)
$$

Where,

$$
r_{i}(t)=\frac{1}{\sqrt{N}} \sum_{m=1}^{N} \cos \left(2 \pi \mathcal{F}_{d} \cos \alpha_{m} t+a_{m}\right)
$$

and

$$
r_{q}(t)=\frac{1}{\sqrt{N}} \sum_{m=1}^{N} \sin \left(2 \pi \mathcal{F}_{d} \cos \alpha_{m} t+b_{m}\right)
$$

$\alpha_{m}$ is the angle of arrival, $a_{m}$ and $b_{m}$ are random phases distributed over 0 to $2 \pi, N$ is number of propagation paths. We have considered only positive Doppler spread in our study to minimize number of sinusoids.

\subsection{BER versus SNR under doubly selective channel}

This section provides the simulation results in-terms of BER performance for the provided SNR under varying channel conditions and various Wavelet bases [11]. Following steps are involved in a single iteration of simulation after initializing channel parameters shown in table I.

- Following are the steps involved in polting BER vs SNR, 
Table 1 Simulation Parameters

\begin{tabular}{lll}
\hline Specification & OFDM & WOFDM \\
\hline No. of Subcarriers & 64 & 64 \\
Modulation Order-QAM & 16 & 16 \\
Subcarrier Spacing & $15 \mathrm{kHz}$ & $15 \mathrm{kHz}$ \\
Carrier Frequency & $2.5 \mathrm{gHz}$ & $2.5 \mathrm{gHz}$ \\
No. of Symbols & 14 & 20 \\
Cyclic Prefix length & $4.76 \mu \mathrm{s}$ & - na- \\
\hline
\end{tabular}

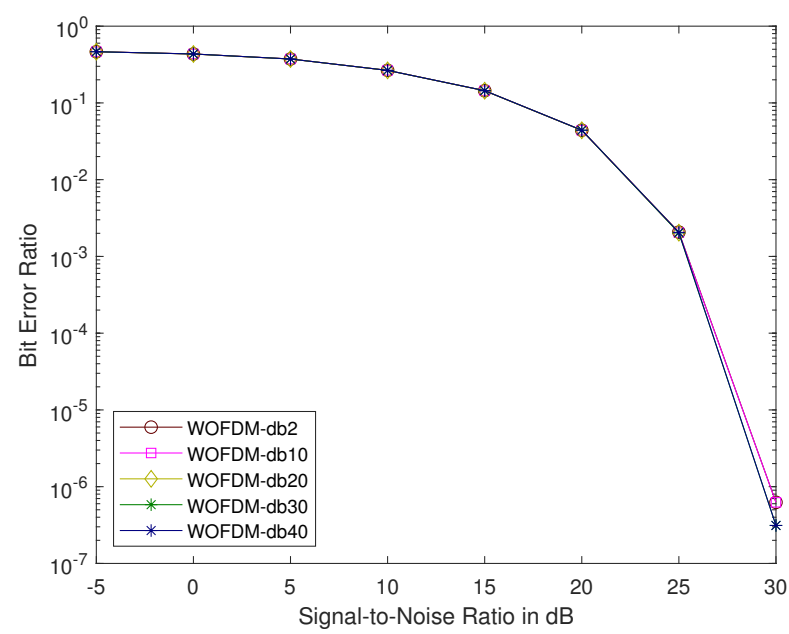

Fig. 2 BER vs. SNR with 0ns delay spread with Daubechies Wavelets

1. Generate random binary bits depending on set channel parameters.

2. Perform Mapping (16-QAM).

3. Add CP to OFDM.

4. Generate Transmit Matrix/Time Domain (IFFT for OFDM and IDWT for WOFDM).

5. Pass transmit matrix data through channel (Convolution).

6. Add Channel Noise.

7. Demodulate Signal/Frequency Domain (FFT for OFDM and DWT for WOFDM).

8. Perform One Tap equalization.

9. Convert symbols to bits (De-Mapping).

10. To calculate the BER $\frac{E_{b}}{N_{0}}$ for the iteration, compare the obtained bits to the corresponding transmitted bits.

11. Repeat Monte-Carlo simulation to take average and plot.

Figure 2 shows the BER of Daubechies ( $\mathrm{db}$ ) wavelet bases with different filter length (db2, db10, db20, db30 and db40) with power delay profile of Additive White Gaussian noise and uniform Doppler spread resulting in 0ns Delay Spread has been averaged over 100 iterations. It is found that 


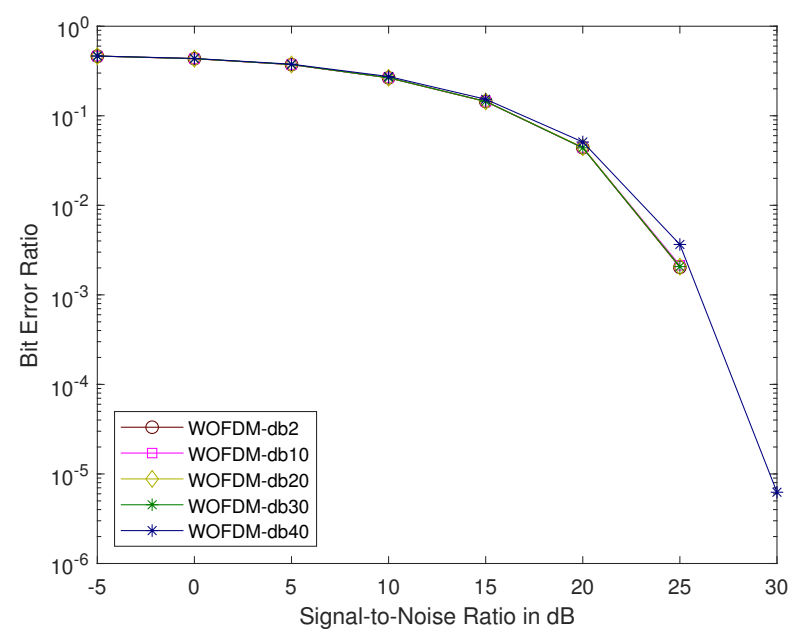

Fig. 3 BER vs. SNR with 0ns delay spread with different Wavelets

Daubechies wavelets with different filter size exhibit almost similar performance of about 1 bit error in every $10^{7}$ bit transmitted at 30 SNR under given channel conditions. Note that, since Cyclic Prefix is not required in WOFDM, same bandwidth is utilized to achieve higher data rates.

Figure 3 shows the BER of different wavelet bases like Daubechies, Symlets, Fejér-Korovkin, Coiflets and Bi-Orthogonal wavelets with fixed filter length (db20, sym16, fk18, coif5 and bior6.8) with power delay profile of Additive White Gaussian noise and uniform Doppler spread with mobility between transmitter and receiver being 0kmph, resulting in 0ns Delay Spread has been averaged over 100 iterations. It is found that different wavelets, except BiOrthogonal with given filter size exhibit almost similar performance of about 1 bit error in every $10^{7}$ bit transmitted at 30 SNR under given channel conditions.

Figure 4 and 5 shows the simulated performance averaged over 100 iterations of Daubechies wavelets with different filter lengths and Different Wavelets with fixed filter lengths respectively. The Power Delay Profile selected for this simulation is 'Pedestrian', mobility is 20kmph and Jake's Doppler spread is chosen for which the calculated delay is $42 \mathrm{~ns}$. In this scenario, the performance of WOFDM is considerably less ( 1 bit error for every 2 bit transmitted). A Daubechies-20 basis is comparatively better by $1 \mathrm{db}$ compared to other wavelet bases. Similar results are found in Figure 6 and 7 in which, PDP selected is 'Vehicular', mobility is $120 \mathrm{kmph}$, and chosen channel Doppler model is 'Uniform' for which the delay calculated is $360 \mathrm{~ns}$.

Finally, figure 8 and 9 gives the comparison of WOFDM with different wavelet basis and OFDM with Cyclic Prefix in 'Pedestrian' and ' Vehicular' power delay profiles and averaged over 100 iterations. It is evident from the result that OFDM with $\mathrm{CP}$ shows better resistance towards doubly selective 


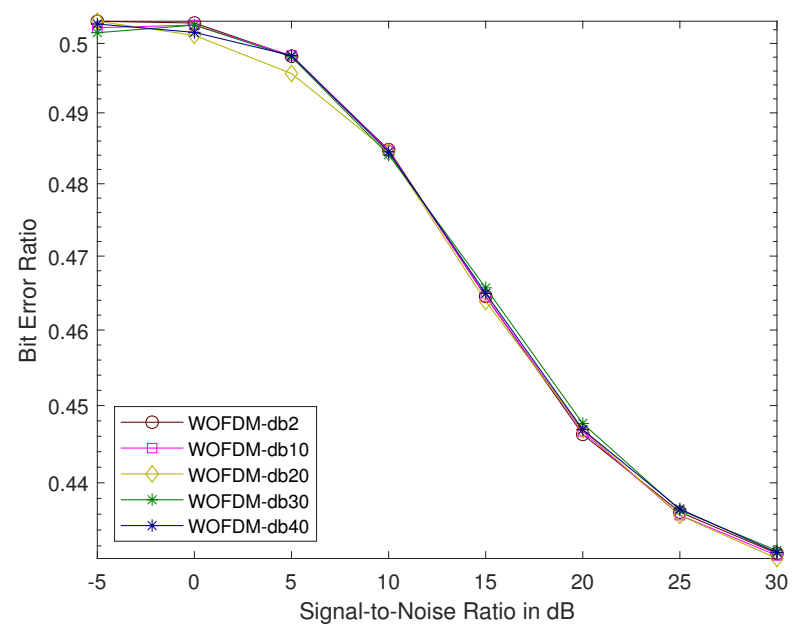

Fig. 4 BER vs. SNR with 42 ns delay spread with Daubechies Wavelets

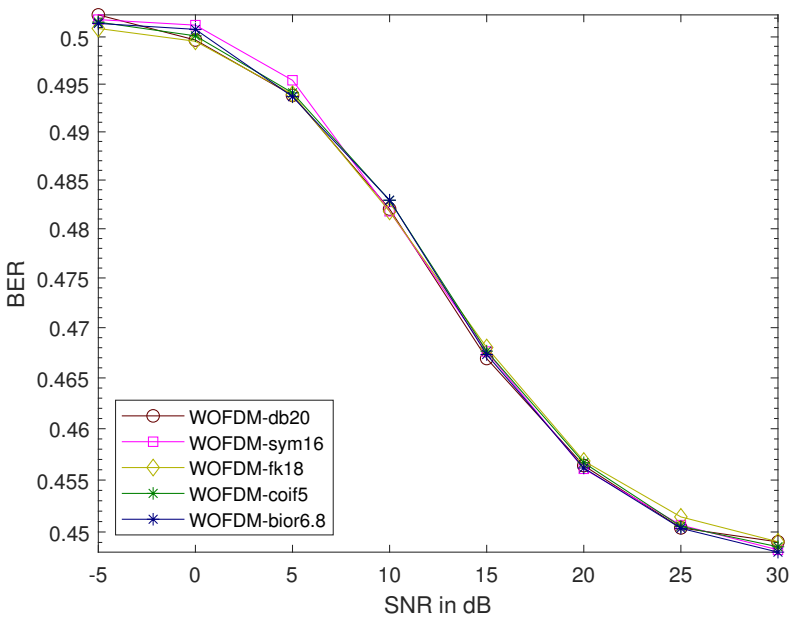

Fig. 5 BER vs. SNR with 42 ns delay spread with different Wavelets

channels. OFDM with CP BER is 1 error in every $10^{3}$ bits transmitted while WOFDM decodes 1 bit error for every alternate bits transmitted.

\subsection{SIR versus Time-Frequency Offset under doubly selective channel}

This section contains simulation results in which the signal-to-interference ratio is determined for time and frequency offsets with various Wavelet bases and compared to Cyclic Prefix OFDM. Following the initialization of channel 


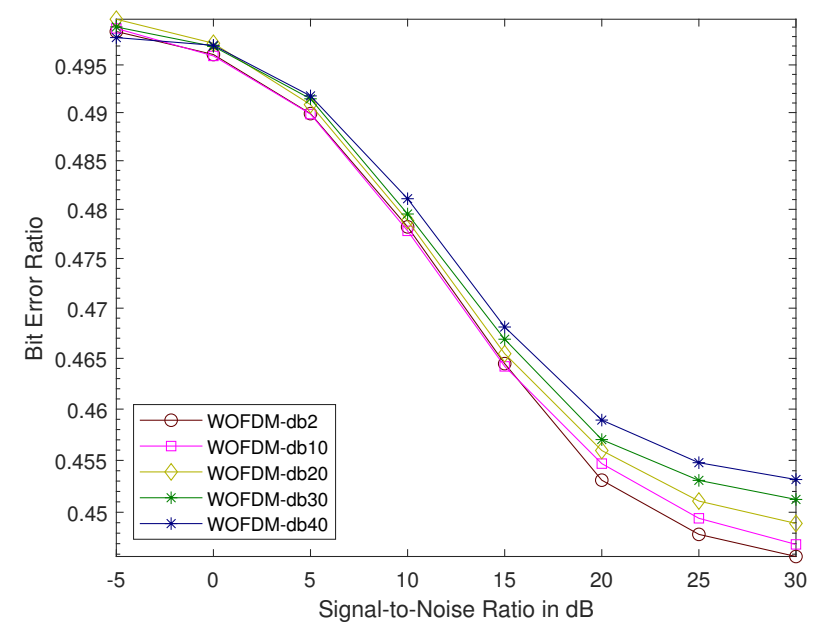

Fig. 6 BER vs. SNR with 360ns Delay Spread

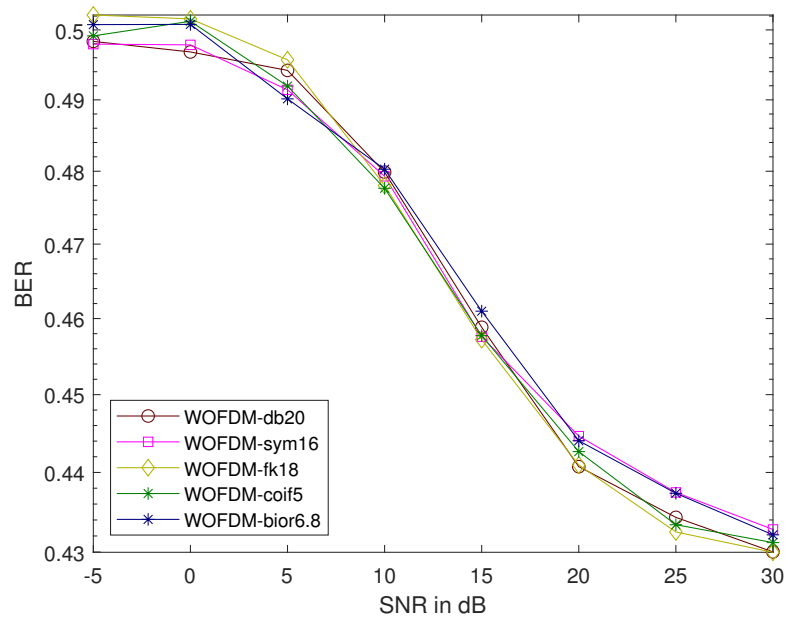

Fig. 7 BER vs. SNR with 360ns Delay Spread

parameters (shown in table I), the following steps are involved in a single simulation iteration.

- Following are the steps involved in claculating SIR for the given offset,

1. Depending on the channel parameters, initialize modulation objects.

2. Generate transmit and receive Matrix.

3. Perform Time Offset and Frequency Offset (Circular shift).

4. Calculate signal to interference ratio.

5. Repeat step 1 to 4 to plot SIR vs. Time-Frequency Offset. 


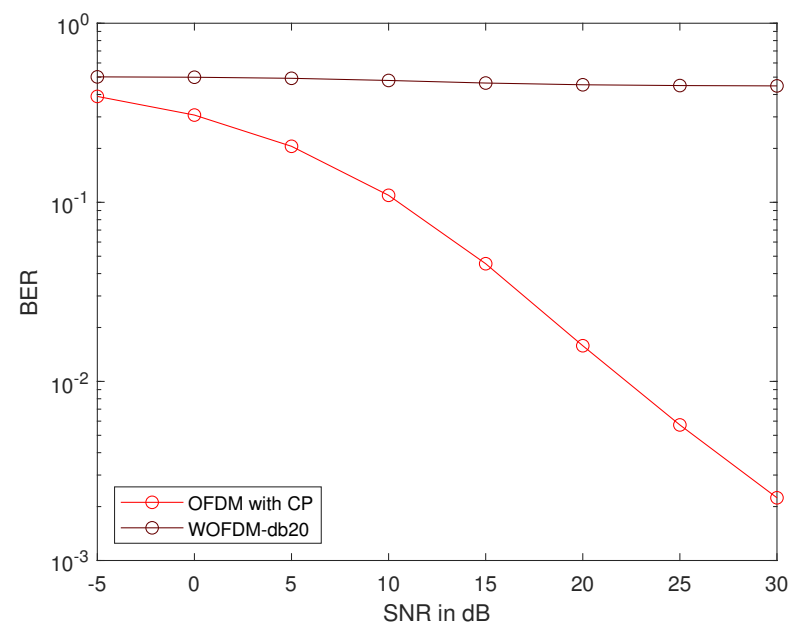

Fig. 8 BER vs. SNR with 360ns Delay Spread

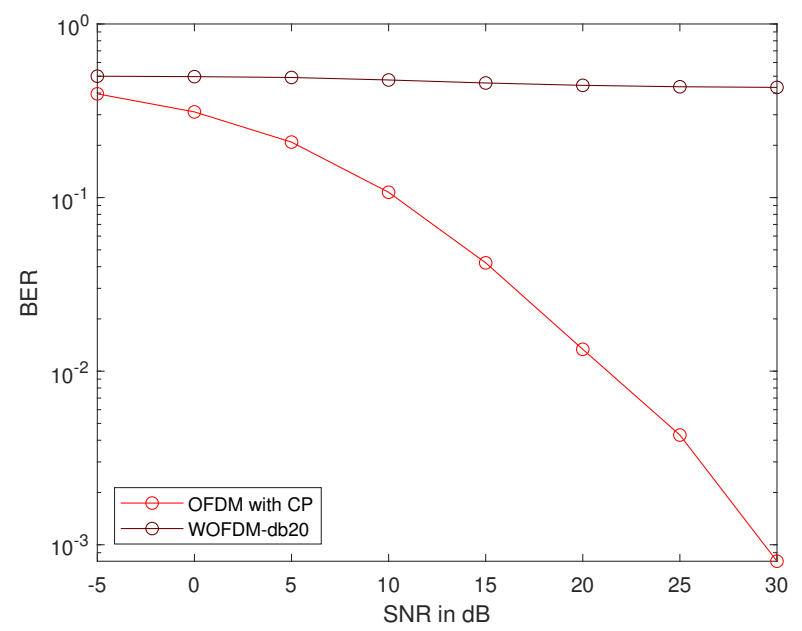

Fig. 9 Figure 9. BER vs. SNR with 42ns Delay Spread

Figure 10 and 12 shows the Signal to Interference Ratio (SIR) vs. frequency offset response of Daubechies wavelet basis with different filter lengths (db2,db10, db20, db30 and db40) and different wavelet basis with fixed filter lengths(db20, sym16, fk18, coif5 and bior6.8). 10 percent normalized Frequency offset/drift shows nearly $5 \mathrm{db}$ improved performance in Daubechies wavelet with filter coefficient of 40 compared to any other wavelet basis. However FFT based OFDM with CP performs better than any compared wavelet basis. Similar wavelet configuration is applied to calculate and plot SIR vs. normalized Time Offset and found that out of applied wavelet basis db20 per- 


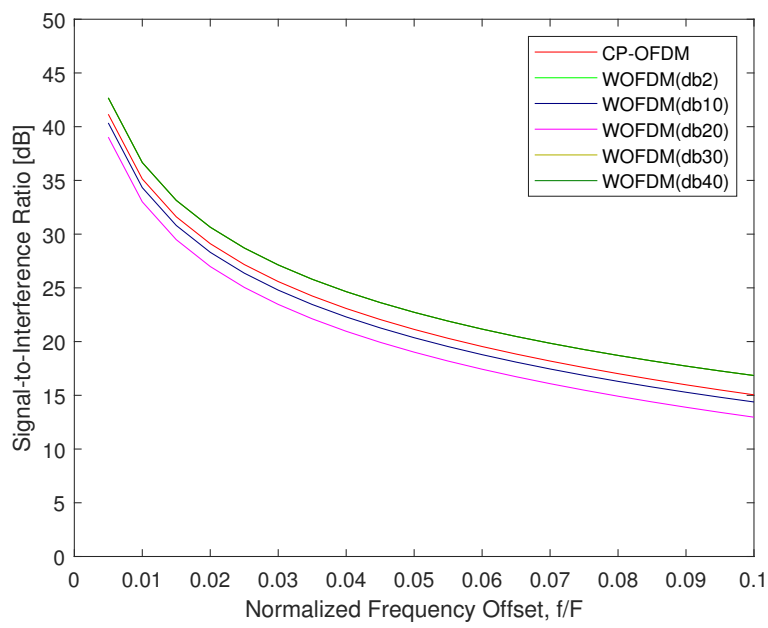

Fig. 10 SIR vs. Frequency Offset of Daubechies Wavelets

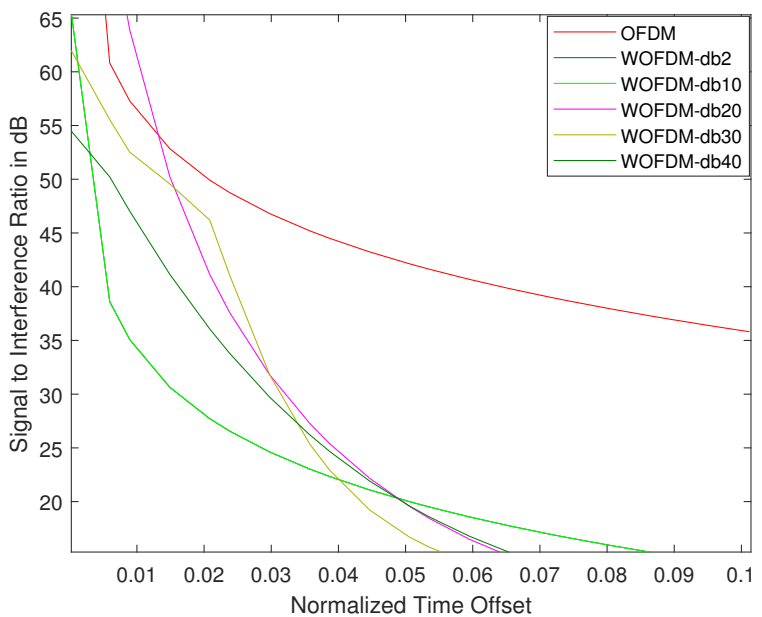

Fig. 11 SIR vs. Time Offset of Daubechies Wavelets

forms better than $4 \mathrm{~dB}$ for nearly 2 percent time offset compared to other wavelet basis, but OFDM with CP outperforms WOFDM by nearly $35 \mathrm{db}$. Table II provides the comparison of numerical results of calculated SIR for the given Time Offset. 


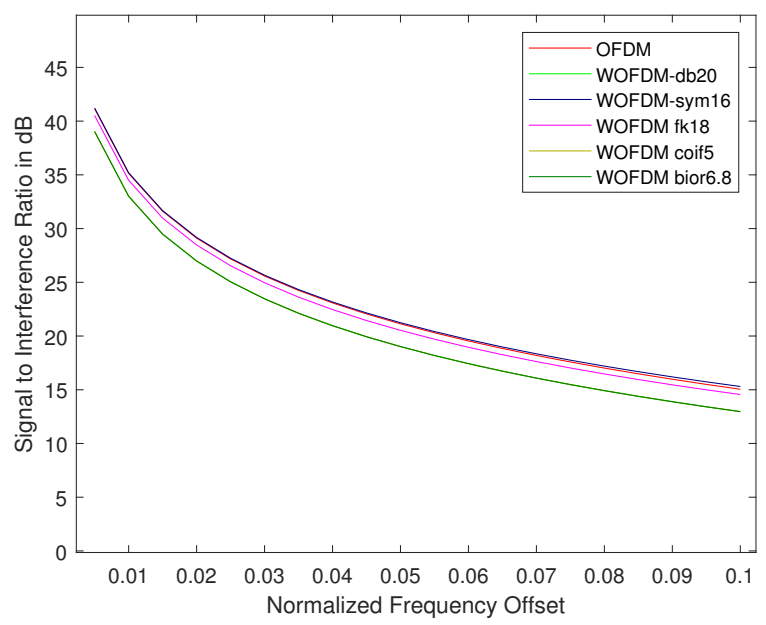

Fig. 12 SIR vs. Frequency Offset of different Wavelets

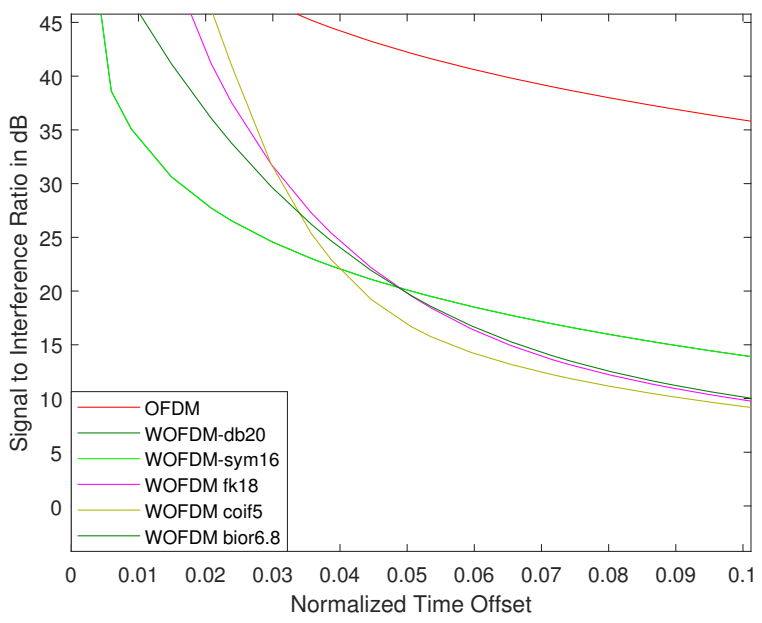

Fig. 13 SIR vs. Time Offset of different Wavelets

\section{Conclusion}

In this paper, we have analyzed and compared the performance of Wavelets Basis in Multi Carrier Modulation Communication systems or simply WOFDM with the FFT based OFDM with Cyclic Prefix jointly under doubly selective fading channel resulting in Time and Frequency Synchronization Errors. It is evident from the results that FFT based CP-OFDM shows considerable resistance to Synchronization error under time and frequency offsets, mainly because of the Cyclic Prefix. On the other hand, Wavelets capability lies in its 
Table 2 Comparison of Calculated SIR in dB with Time Offset for various Basis

\begin{tabular}{lllllll}
\hline Time Offset in \% & 0 & 2 & 4 & 6 & 8 & 10 \\
\hline FFT (OFDM with CP) & 103 & 65 & 50 & 46 & 43 & 40 \\
db2 & 64 & 25 & 21 & 18 & 14 & 9 \\
db10 & 66 & 24 & 21 & 19 & 15 & 12 \\
db20 & 75 & 31 & 26 & 19 & 15 & 12 \\
db30 & 70 & 30 & 20 & 19 & 13 & 12 \\
db40 & 68 & 29 & 19 & 17 & 13 & 11 \\
sym16 & 60 & 26 & 23 & 21 & 19 & 15 \\
fk18 & 65 & 31 & 21 & 18 & 15 & 12 \\
coif5 & 55 & 26 & 20 & 17 & 14 & 10 \\
bior6.8 & 60 & 29 & 25 & 20 & 15 & 12 \\
\hline
\end{tabular}

adaptability, which is very much necessary in Cognitive radio systems. This paper considers the readily available off the shelf wavelets, which shows relatively higher capacity as $\mathrm{CP}$ is not required and good provides BER under ideal channel conditions. However, slightest change in the channel conditions results in a severe degradation in the performance due to loss of orthogonality. Out of six wavelet basis used, Daubechies-20 (db20) performs better compared to others but at the cost of computational efforts due to higher filter coefficients. Wavelets specifically designed to match the channel conditions are required, which may show strong resistance to channel impairments due to time or frequency.

Acknowledgements I Would like to thank my research guide Dr. Surekha T P for her support and guidance.

\section{Declarations}

\section{Conflict of interest}

The authors declare that they have no conflict of interest.

\section{Funding}

The author(s) received no specific funding for this work.

\section{Availability of Data}

Not Applicable.

\section{Code Availability}

Not Applicable. 


\section{References}

1. Abdzadeh-Ziabari, H., Zhu, W.P., Swamy, M.: Joint maximum likelihood timing, frequency offset, and doubly selective channel estimation for ofdm systems. IEEE Transactions on Vehicular Technology 67(3), 2787-2791 (2017)

2. Abdzadeh-Ziabari, H., Zhu, W.P., Swamy, M.: Timing and frequency synchronization and doubly selective channel estimation for ofdma uplink. IEEE Transactions on Circuits and Systems II: Express Briefs 67(1), 62-66 (2019)

3. Baghaki, A., Champagne, B.: Joint frequency offset, time offset, and channel estimation for ofdm/oqam systems. EURASIP journal on advances in signal processing 2018(1), 1-19 (2018)

4. Chafii, M., Palicot, J., Gribonval, R., Bader, F.: Adaptive wavelet packet modulation. IEEE Transactions on Communications 66(7), 2947-2957 (2018)

5. Chin, W.L., Chen, S.G.: Joint effects of synchronization errors of ofdm systems in doubly-selective fading channels. EURASIP Journal on Advances in Signal Processing 2008, 1-9 (2008)

6. Chin, W.L., Kao, C.W., Chen, H.H., Liao, T.L.: Iterative synchronization-assisted detection of ofdm signals in cognitive radio systems. IEEE transactions on vehicular technology 63(4), 1633-1644 (2013)

7. Domínguez-Bolaño, T., Rodríguez-Piñeiro, J., García-Naya, J.A., Castedo, L.: Bit error probability and capacity bound of ofdm systems in deterministic doubly-selective channels. IEEE Transactions on Vehicular Technology 69(10), 11458-11469 (2020)

8. Guo, W., Li, C., Zhao, H., Wen, R., Tang, Y.: Comprehensive effects of imperfect synchronization and channel estimation on known interference cancellation. IEEE Transactions on Vehicular Technology 69(1), 457-470 (2020). DOI 10.1109/TVT.2019.2950046

9. Jamin, A., Mähönen, P.: Wavelet packet modulation for wireless communications. Wireless Communications and Mobile Computing 5(2), 123-137 (2005)

10. Kumar, M., Majhi, S.: Joint signal detection and synchronization for ofdm based cognitive radio networks and its implementation. Wireless Networks 25(2), 699-712 (2019)

11. Kumar, S., Chaudhari, M.S., Gupta, R., Majhi, S.: Multiple cfos estimation and implementation of sc-fdma uplink system using oversampling and iterative method. IEEE Transactions on Vehicular Technology 69(6), 6254-6263 (2020)

12. Lakshmanan, M.K., Karamehmedović, D., Nikookar, H.: An investigation on the sensitivity of wavelet packet modulation to time synchronization error. Wireless Personal Communications 58(3), 483-502 (2011)

13. Mitola, J., Maguire, G.: Cognitive radio: making software radios more personal. IEEE Personal Communications 6(4), 13-18 (1999). DOI 10.1109/98.788210

14. Nasir, A.A., Durrani, S., Mehrpouyan, H., Blostein, S.D., Kennedy, R.A.: Timing and carrier synchronization in wireless communication systems: a survey and classification of research in the last 5 years. EURASIP Journal on Wireless Communications and Networking 2016(1), 1-38 (2016)

15. Nissel, R., Schwarz, S., Rupp, M.: Filter bank multicarrier modulation schemes for future mobile communications. IEEE Journal on Selected Areas in Communications 35(8), 1768-1782 (2017)

16. Sharma, S.K., Bogale, T.E., Chatzinotas, S., Ottersten, B., Le, L.B., Wang, X.: Cognitive radio techniques under practical imperfections: A survey. IEEE Communications Surveys \& Tutorials 17(4), 1858-1884 (2015)

17. Wilson, K., Wilson, S., Biglieri, E.: Academic press library in mobile and wireless communications (2016)

18. Xiao, C., Zheng, Y.R., Beaulieu, N.C.: Second-order statistical properties of the wss jakes' fading channel simulator. IEEE Transactions on Communications 50(6), 888-891 (2002)

19. Xie, X., Peng, M., Li, Y., Wang, W., Poor, H.V.: Channel estimation for two-way relay networks in the presence of synchronization errors. IEEE Transactions on Signal Processing 62(23), 6235-6248 (2014) 


\section{AUTHORS PROFILE}

Arjun K R earned his B.E. in Electronics and Communication in 2009 and his M.tech in Digital Electronics and Communication in 2011. He is currently working on his $\mathrm{PhD}$ in Wireless Communication. He has published papers in national and international journals. He is currently a research scholar at Vidyavardhaka College of Engineering.

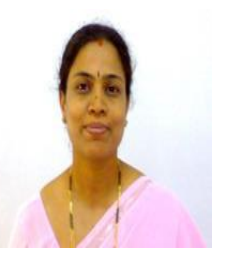

Dr. T P Surekha earned her bachelor's and master's degrees in ECE and Bio Medical Signal Processing, respectively, in 1990 and 2000. In the year 2013, she completed her doctorate in the discipline of communication systems. She has extensive teaching and research expertise. She is currently employed as an ECE professor at Vidyavardhaka College of

Engineering in Mysuru, Karnataka, India. She has 45 papers in national and international publications and conferences to her credit. Wireless communication, microwave antennas, and biomedical signal processing are among her research interests. I.S.T.E., Institute of Engineers (India), and IETE India are all life members of the author. 